\title{
Study of V2G applications in residence community and parking lot
}

\author{
Ranchen Yang ${ }^{1, a}$, Guojing Dong ${ }^{1, b}$,Chaoqun Sheng ${ }^{1, c}$ \\ North China Electric Power University, Baoding, 071000, China.

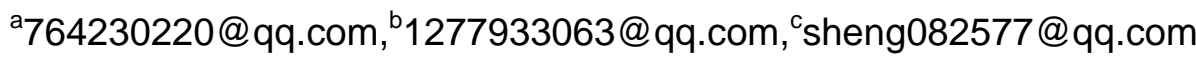

Keywords: Electric vehicles, Order charging, V2G, Game model.

\begin{abstract}
With the thoroughly implementation the of the concept of sustainable development, the electric cars become more and more popular, and as the increasing scale of mobile storage devices, electric vehicles, its ordered charge and discharge is playing an extremely important role in the smooth operation of the power grid. Therefore, studying the V2G running mode of electric vehicles and thenworking out concrete and workable programs is important. This paper, aimed at community residents and parks, proposes specific operational programs based on the existing researches, and demonstrates the effects of ordered charging on peak and off-peak load. In addition, this paper aims to solve some specific operational issues, such as the charging in stations and the vehicles management, which has laida solid foundation for V2G model to put into operation and make a better promotion in future.
\end{abstract}

\section{Introduction}

On the research aboutthe effect of electric vehicles charging on the power grid, the references [1-3], focuses on analyzingthe impact of a large-scale electric vehiclesconnected to power grid, in particular, makesa detailed analysis of harmonic influence.It carries out charging load simulation model from different angles andcome up with method of weakening harmonic pollution based on the simulation results. The reference [4] constructs response model for users to select the charging time during the period of peak-Valley electricity. Then, based on the practical load situation of power grid underthe simulation of forming Monte Carlo, it discusses the effect of ordered charging on the power grid to achieve the shifting peak to valley load.

In terms of operation mode of electric vehicles charging stations, according to the abroad actual conditions, owing to the differences existing between technologies and ways of charging, the operating modes of electric vehicles charging stations can be divided into two modes: "vehicle charging" and "battery replacement". Vehicle charging mode regards the battery and the vehicle as an entirety.The key for its large-scale development is to develop and produce "large capacity, low cost, fast charging, long product life" batteries. Battery replacement mode is also known as leasing the battery model, which is considered separately from the battery and vehicle. Users only need to buy cars, and a specialized leasing the battery companies charge the purchase of batteries, leasing, charging, fast-exchange and management.

This paper, based on project, through the investigation of specific situation about using and idling electric vehicles, combined with a large number of research data and literature at home and abroad about electric vehicles charging on station, aims to improve the V2G model specific operating mechanism based on residential area and car parks.

\section{Analysis of user behavior}

At present, in large cities, due to the centralized distribution of the residential and working areas, "Chao-Sha" phenomenon is known as the urban transportation features. "Chao-Sha" refers to that a large number of urban residents go along the same direction of travel during rush hour on work, while a lot of people go back in the opposite direction during the rush hour when they get off work. Taking the results of the fourth comprehensive traffic investigation of Shanghai Municipal Government as an example, we analyzethe vehicles using behavior and law of urban private car users in the following aspects. 


\subsection{The travel purposes of car users}

Traffic in the city according to the different travel purposes can be divided into commutingand non-commuting. Commuting refers to the travel between residence and work place (including work, school, and corporate). Commuting is a way that travel route and round-trip time are relatively fixed. Commuting occupies a larger proportion in the urban transport.

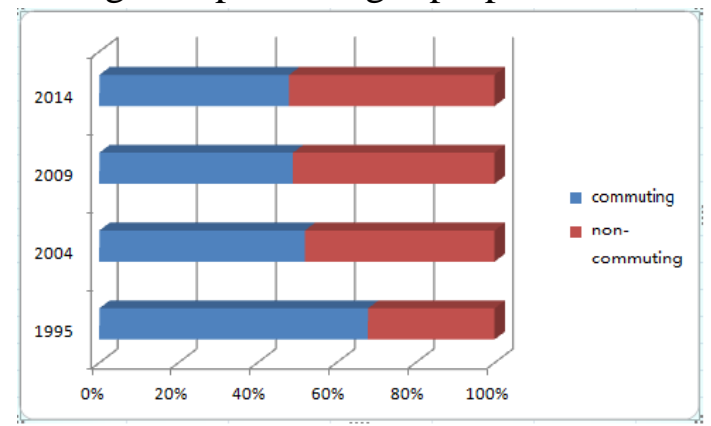

Figure 1. The proportion of cars for different purposes

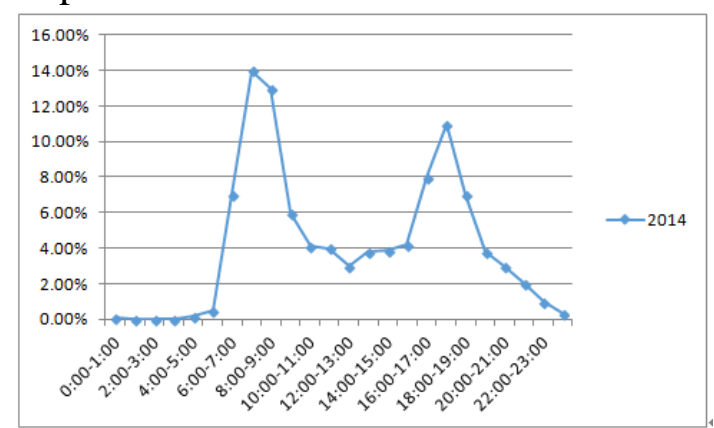

Figure 2. Distribution of travel time by car

Non-commuting including: going out for entertainment, travel such as visit relatives, shopping and entertainment. Compared to commuting, its travel route and time is more random. Whether commuting or non-commuting, all shows a shorter path transportation within a period of 24 hours, which reflects the vast majority of car users' behavior in the city.

\subsection{Travel time}

Figure 2 shows the travel proportion of the private users in the 24 hours a day (on a per-hour intervals). As can be seen from the figure, in a day,the time for travel of car usersmainly distribute at 5:00-22:00, of which 6:00-10:00 and 16:00-20:00 are the most concentrated periods of the day, taking up $70 \%$ of traffic in a day or so. Through the analysis of travel time distribution, we can infer the law of battery consumption about the electric vehicle users in the course of a day's ride, thus we can predict the regularity of change about the needs of the electric vehicle charging in the city over time, which has an important guiding significance for charging network planning.

2.3 parking

In General, the ideal way for electric vehicles to charge is making use of the time during the parking. We can see from Figure 2, the vast majority of civilian vehicles are in the docked state during 22:00-6:00 in the city. According to the analysis, during this time, users tend to choose the standard charge by using charge-pile. Figure 4 shows that the ways of users selected parking at night and the proportion. Among them, the Community construction of parking spaces takes up $60 \%$, accounted for the majority. Because of the low conditions of the standard charge-pile construction, the residential parking construction, public parking spaces all can be constructed. Both the two parking modes account for $68 \%$. It can be assumed that we can basically meet the needs of the users of this electric vehicles through the construction of a sufficient number of standard charging-pile.

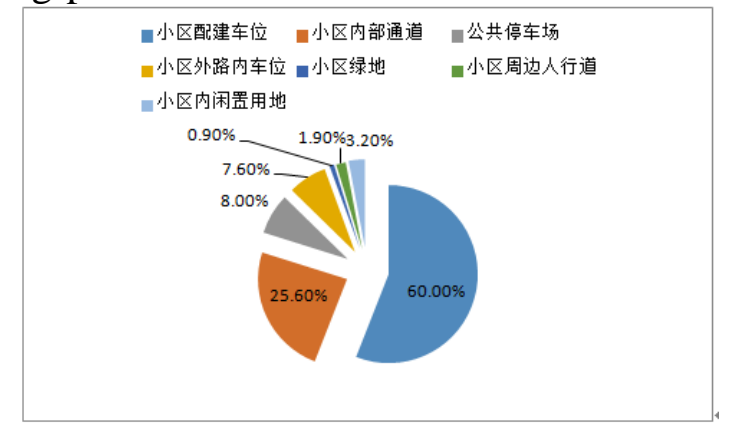

Figure 3. The proportion of car park ways

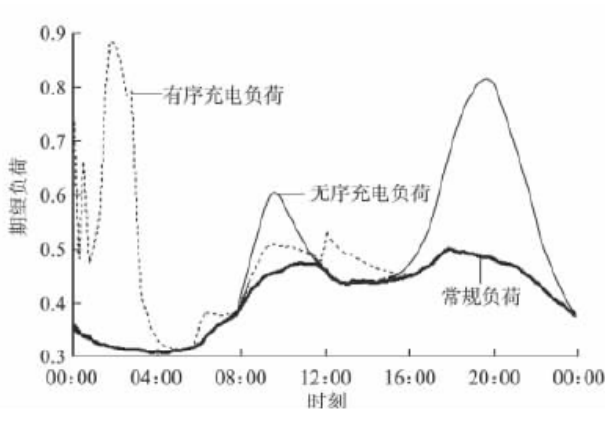

Figure4. The ordered charges and disordered charges 


\section{Simulating the daily load demand of electric cars}

Assume private cars take a single and a one-time full charge each day.Initial SOC meet the normal distribution:

$$
\text { SOC }=\frac{1}{\sqrt{2+0.2 \pi}} e^{-\frac{(X-0.6)}{2+0.2^{2}}}
$$

The current of regular and fast charge is $0.2 \mathrm{C}$ and $1.25 \mathrm{C}$ respectively, the battery charging from zero to full separately needs $5 \mathrm{~h}$ and $0.8 \mathrm{~h}$.

By using Monte Carlo to simulatingcharging requirements of 100 vehicles in one day, we count the results ofthe two modes order charges and disorderly charges and make a chart.

Through analyzing the two typical daily load curves of ordered charges and disorderly charge, we discover that under the disorderly charging mode, in the evening load rush hour, a large number of electric vehicles join up charging, which leads to evening peak higher, thus increasing the valley-to-peak. While under ordered charging mode, even though evening peak does not increased further, however, in theevening valley, due to the low price of purchased electricity, for the sake of getting larger economic, the charging station concentratea large number of electric vehicles to charge in this period of time, which leads to a partial electricity peak in night power grid.This electricity peak even higher than the evening peak.This shows that in the situation of quantities of electric vehicles connected to the power grid, there will be a great deal of vehicles gathered at the cheaper electricity price time to charge if we simply use the time of use (TOU)TO regulatethe behavior of charging in the electric charging station, which could lead to the emergence of another partial power peaks.

To curb the appearance of the evening peak, we can increase the number of replaceable batteries, and go to charge part of the batteries needed to be charged in the next day whenthe power consumption is relatively low (approximately 13-16 hours). As for the number of replaceable batteries to be provided, it needs further study.

\section{The concrete operating V2G mode based on the residential area andparking lots}

The V2G mode based on the residential area and parking lotscomes to understand the specific interests requirements of owners involved in the V2G mode operation mainly through research.Through the consultations with the power supply Bureau, they agree with the electricity supply price in the valley andthe return electricity price at the peak, using price differences to compensate the owners.

The specific operating modecan be improved on the available mode. For communities with centralized parking, we count the vehicles'dump energy when they enter the community parking lots.If reached the reserve power, we make centralized processing to connect with the power grid by the V2G mode technology as a backup. After the end of the standby time, in the night-time electricity valley, that is, from 11 o'clock in the evening to 6 o'clock the next day, take centralized charging of vehicles. Under the premise of reaching the reserve capacity, if it is served as the alternate in a row before 11 o'clock in the evening in the parking lot, longer than three hours, we do remember an alternate, monthly count reserve number per vehicle, and distributealternative compensation according to the number.

\section{Conclusion}

The V2G mode theory of electric vehicles is quite perfect, while thepractice exploration never stop.How tofeasibly put into practice is now urgently needed to be solved. This paper,using resident community and parking lots for carrier, simulate day load demand of electric vehicles, and forecast the favorable effect of ordered charging on power grid peak-valley load.In addition, this paper has more targeted to solvesome specific operation problems including the billing of charging station and the vehicles management. But because of limited capacity andstilling staying in academic phase, the specific operations needs further study. 


\section{References}

[1]Liu Qing. The charging mode and the effect of charging stations for electric energy quality analysis [J]. China's high-tech enterprises, 2011,09 (27): 43-45

[2] Huang Mei, Huang Shaofang, Jiang Jiuchun. Electric vehicle charger (station) access the harmonic analysis of power system [J]. Journal of Beijing Jiaotong University, 2008,32 (05): 86-88

[3]Lu Yanxia,Jiang Jiuchun. Harmonic Study of Electric Vehicle Chargers[C]. ICEMS2005: Proceedings of the Eighth International Conference on Electrical Machines andSystems,2005: 2404-2407

[4]Optimization of peak-Valley electricity price ordered electric vehicle charging _ Ge Shaoyun

[5]Kristien Clement-Nyns,Edwin Haesen,Johan Driesen. The Impact of ChargingPlug-In Hybrid Electric Vehicles on a Residential Distribution Grid [J]. IEEETransactions on power systems,2010,25(1): 371-380

[6]Analysis on operation mode of electric vehicle charging stations Zhou Fengquan 1, 1 Lian Zhan Wei, Wang Xiaolei 1,2, Yang Jiaohui 1, Xu Yishan 1 (1. XJ Group Corporation, xuchang, Henan 461000; 2 , Zhongyuan, Henan Zhengzhou 450007) 